Revue

Revue de l'histoire des religions

de Ihistoire des religions

$3 \mid 2018$

Varia

\title{
Anges, réforme et désenchantement du monde dans l'Angleterre moderne
}

Notes critiques

Angels, Reform and the Disenchantment of the World in Early Modern England

Antoine Mazurek

\section{OpenEdition}

Journals

Édition électronique

URL : http://journals.openedition.org/rhr/8964

DOI : $10.4000 /$ rhr.8964

ISSN : 2105-2573

Éditeur

Armand Colin

Édition imprimée

Date de publication : 1 septembre 2018

Pagination : 507-529

ISBN : 978-2-200-93188-9

ISSN : 0035-1423

Référence électronique

Antoine Mazurek, "Anges, réforme et désenchantement du monde dans l'Angleterre moderne », Revue de I'histoire des religions [En ligne], 3 | 2018, mis en ligne le 01 septembre 2020, consulté le 15 janvier 2021. URL : http://journals.openedition.org/rhr/8964 ; DOI : https://doi.org/10.4000/rhr.8964 


\section{Anges, réforme et désenchantement du monde dans l'Angleterre moderne Notes critiques*}

Plusieurs ouvrages récents proposent une étude de la présence des anges dans l'Angleterre des XVI et XVII siècles. Combinant une approche en termes d'histoire sociale et culturelle, ils ont l'ambition d'inscrire les anges dans l'ensemble de la culture anglaise. Ce faisant, la définition même de l'objet " ange » est revisitée et la question du désenchantement, dont la pertinence demeure une préoccupation majeure de l'historiographie anglo-saxonne des réformes, est soumise à un nouvel examen.

\section{Angels, Reform and the Disenchantment of the World in Early Modern England}

Recent studies offer a fresh look at the presence of angels in $16^{\text {th }}$-and $17^{\text {th }}$-century England. Combining a social and a cultural approach, they aim to contextualize angels within the whole of English culture. In so doing, the definition of "angel" is revisited and the question of disenchantment, whose relevance for understanding the Reformation is a major concern for English historiography, is reassessed.

* À propos des trois ouvrages suivants : Joad Raymond, Milton's angels. The early modern imagination, Oxford, Oxford University Press, 2010, XVIII-465 p., 23 cm, $30 £$, ISBN 978-0-19-956050-9; Joad Raymond (éd.), Conversations with angels. Essays towards a history of spiritual communication, 1100-1700, New York, Palgrave Macmillan, 2011, XIII-349, $23 \mathrm{~cm}, 116 €$, ISBN 978-0230-55203-6 ; Laura Sangha, Angels and belief in England, 1480-1700, Londres, Pickering \& Chatto, 2012, IX-265 p., 24 cm, 95 £, ISBN 978-1-84893-145-9. 
Dans le chapitre 34 du Léviathan, Thomas Hobbes analyse la signification dans l'Écriture des termes esprit, ange et inspiration. Sa lecture de la Bible l'amène à confirmer ce que le raisonnement naturel avait déjà mis en évidence :

En examinant la signification du mot ange dans l'Ancien Testament, et la nature des rêves et des visions qui surgissent en nous selon les voies ordinaires de la nature, je fus porté à faire mienne l'opinion selon laquelle les anges ne sont rien que les apparitions surnaturelles des fantaisies de l'imagination, suscitées par l'opération spéciale extraordinaire de Dieu par où il fait connaître sa présence et communique ses commandements au genre humain, et surtout à son propre peuple ${ }^{1}$.

Hobbes admet ensuite la réalité substantielle des anges dans le récit du Nouveau Testament mais avec beaucoup de circonspection : le lecteur est donc largement conforté dans la conviction que les anges ne sont que des illusions dérivées de la sensation et que le terme a dans la Bible un usage métaphorique. La charge contre l'angélologie, qui revient à plusieurs reprises dans l'ouvrage, est d'abord une charge contre les absurdités attribuées à la théologie scolastique. Les spéculations sur les anges sont en effet ce qui illustre le mieux cette «vaine philosophie» que Hobbes dénonce tout au long de son livre. Mais les anges, présentés par les théologiens comme des substances incorporelles prenant des apparences sensibles pour communiquer avec les hommes, ne sont pas seulement la cible du matérialisme du philosophe anglais. Ils sont également un défi lancé à sa théorie politique dans la mesure où, avec les prophéties et les inspirations, ils constituent des concurrents directs du pouvoir politique auquel il veut donner un pouvoir absolu, y compris dans le domaine religieux - c'est-àdire plus précisément dans l'interprétation de l'Écriture. En cela, la critique de la réalité des anges est aussi une réponse au contexte d'effervescence religieuse du milieu du XVII ${ }^{\mathrm{e}}$ siècle anglais où la multiplication des écrits sur les anges et les esprits est considérée par Hobbes comme un symptôme et même une cause de la guerre

1. Thomas Hobbes, Léviathan ou Matière, forme et puissance de l'État chrétien et civil, trad. Gérard Mairet, Paris, 2000, p. 581 [1 $1^{\mathrm{re}}$ édition anglaise, Londres, 1651]. 
civile qui embrase les Îles britanniques. Cette critique radicale a immédiatement suscité des réponses visant à défendre, en même temps que les anges, la religion chrétienne. On devine alors l'intérêt que peut susciter une étude des anges dans l'Angleterre moderne. Leur présence dans des pans entiers de la culture anglaise du XVII ${ }^{\mathrm{e}}$ siècle interroge en effet à la fois les modalités de la réforme protestante en Angleterre et, au-delà, la validité du paradigme du désenchantement qui lui est appliqué avec plus ou moins de vigueur.

\section{L'HISTORIOGRAPHIE DE LA RÉFORME ANGLAISE ET LA QUESTION DU DÉSENCHANTEMENT}

Ces recherches s'inscrivent d'abord dans l'historiographie « révisionniste » de la Réforme anglaise qui s'est affirmée dans les années 1980 avec notamment les travaux d'Eamon Duffy et Christopher Haigh et qui se prolonge largement aujourd'hui dans une historiographie qualifiée de post-révisionniste. On peut y distinguer deux idées-force. L'une serait que les Anglais sont devenus protestants plus tard qu'on ne le pensait jusqu'à présent; l'autre, intimement liée à la première, que la religion tardo-médiévale se caractériserait plus, au moment des premières mesures de la réforme henricienne, par sa vitalité que par un déclin irrémédiable ${ }^{2}$. À ces deux idées s'adjoint une approche qui envisage les croyances et les pratiques moins en termes de résistance aux changements idéologiques et doctrinaux que d'assimilation et de transformation. Il y aurait eu, durant le siècle postérieur au schisme, fusion progressive du neuf et de la tradition dans un «patchwork de croyances » typiquement «postreformation » mais pas complètement protestant ${ }^{3}$. La chronologie

2. Voir Eamon Duffy, The stripping of the altars: traditional religion in England, c. 1400-1580, New Haven, 1992 ; Christopher Haigh (éd.), The English Reformation revised, Cambridge, 1987. Sur cette historiographie, voir E. Duffy, «The English Reformation after revisionism», Renaissance Quarterly, 59/3, 2006, p. 720-731 et Peter Marshall, «(Re)defining the English Reformation», Journal of British Studies, 99/1, 2009, p. 564-586.

3. Voir l'introduction de Laura Sangha, Angels and belief in England, 1480-1700, Londres, 2012, qui cite Tessa Watt, Cheap print and popular piety, 1550-1640, Cambridge, 1991, p. 327. 
a en conséquence été modifiée. Alors que l'historiographie traditionnelle estimait que la Réforme entamée sous Henri VIII s'était conclue avec le Règlement élisabéthain de 1559-1563, le révisionnisme parle au contraire d'une «longue Réformation » en la dilatant au point d'embrasser parfois l'ensemble de la période moderne, lorgnant même parfois vers l'époque ultérieure ${ }^{4}$.

Un second courant historiographique forme la toile de fond de ces études, celui qui interroge le rôle de la Réforme dans le processus de modernisation de la culture européenne. Cette question, controversée s'il en est, a suscité une masse d'études relatives aux différents aspects du surnaturel. Depuis le livre de Keith Thomas, Religion and the decline of magic paru en 1971, qui, sans le reconnaître explicitement, reprend à Weber le paradigme d'un «désenchantement du monde », la Réforme est créditée d'avoir donné naissance à un monde moderne sécularisé débarrassé d'une vision magique du monde et de l'immanence du sacré. Or ce paradigme est, comme celui de la rupture franche provoquée par la réforme anglaise, largement remis en cause par l'historiographie récente au point que cette remise en cause est devenue à son tour l'objet d'un large consensus 5 . Pour se confronter à des problématiques aussi générales, la recherche ne prétend plus embrasser les vastes horizons étudiés par Keith Thomas. Elle s'est fragmentée en s'attachant à des aspects particuliers censés évaluer la vitesse et l'extension de ces changements culturels et confronter le discours des réformateurs aux pratiques des populations passées à la Réforme : les attitudes face à la mort, la conception de la providence, les miracles et les anges.

\section{LES ANGES ENTRE RUPTURE ET CONTINUITÉ : SUBSTITUTION, RÉSIDU, ACCOMMODATION, ADAPTATION}

C'est l'ouvrage collectif dirigé par Alexandra Walsham et Peter Marshall qui a ouvert la voie en 2006 en envisageant la question, à l'échelle du monde, des premiers réformateurs jusqu'à

4. Voir par exemple Nicholas Tyacke (éd.), England's long Reformation, 1500-1800, Londres, 1998.

5. Alexandra Walsham, "The Reformation and "the disenchantment of the world" reassessed », The Historical Journal, 51/2, 2008, p. 497-528. 
la fin du XVIII ${ }^{\mathrm{e}}$ siècle ${ }^{6}$. Les hypothèses ont été depuis approfondies autant par ces deux auteurs, qui comptent parmi les historiens les plus influents et prolifiques de la réforme anglaise, que par leurs étudiants et collègues. Nous nous arrêterons plus particulièrement, dans ces notes critiques, sur trois ouvrages. Le premier constitue à la fois une magistrale mise en contexte et une relecture du chefd'œuvre de la poésie anglaise du XVII ${ }^{\mathrm{e}}$ siècle, Le Paradis perdu de John Milton où la présence des anges est singulièrement forte. Le deuxième est issu d'une thèse de doctorat soutenue à l'université de Warwick en 2009 sous la direction de Peter Marshall et envisage la place des anges au sein des croyances en Angleterre. Le troisième est un recueil d'études intitulé Conversations avec les anges qui, par les bornes chronologiques choisies, 1100-1700, la diversité des terrains envisagés et des approches adoptées, manifeste à la fois la volonté de situer la figure dans l'ensemble d'une culture - celle de l'Europe occidentale - et la conviction que la prise en compte d'un temps long est mieux à même d'en dévoiler la signification, quitte à apprécier ce temps long à partir des ruptures dont il est parsemé.

Le même constat est fait d'emblée: les anges sont partout dans l'Angleterre d'après la Réforme. On peut se demander assez naïvement comment il est possible de mesurer une telle ubiquité. La réponse n'est pas aussi simple qu'il y paraît car elle met en question la méthode de l'histoire culturelle. C'est en effet un exercice difficile que de jauger la place d'une croyance comme celle aux anges dans l'ensemble d'une culture. Raymond comme Sangha fondent leur constat sur la profusion des mentions des anges dans «tous les types d'écrit» de l'époque ${ }^{7}$ tout en admettant par ailleurs que le traitement frontal de l'angélologie est marginal dans les ouvrages du XVII ${ }^{e}$ siècle et même très discret dans les textes officiels et les catéchismes, l'ubiquité n'étant pas synonyme de centralité8. Pour en dégager la signification, il est donc nécessaire de prêter attention au contexte d'énonciation - social, politique - car il a des effets sur

6. P. Marshall et A. Walsham (éd.), Angels in the early modern world, Cambridge, 2006.

7. Joad Raymond, Milton's angels. The early modern imagination, Oxford, 2010, p. 20 : «Angels penetrate all kinds of writing in sixteenth- and seventeenthcentury Britain ».

8. L. Sangha, Angels and belief, p. 95, 146. 
la doctrine. Joad Raymond remarque ainsi que ces divers registres d'écriture «ne reflètent pas simplement un corpus préexistant de pensées » mais « interagissent avec les notions d'être et d'action angélique $»^{9}$.

L'ubiquité angélique a un fondement théorique, celui qui avait rendu et qui rendait encore possible dans le domaine catholique l'inflation de l'angélologie, à savoir la grande «malléabilité » des anges, qui en fait une sorte d'auberge espagnole de la théologie, un simple « outil rhétorique », un «motif» voire un «espace culturel » à même de recevoir tous les contenus de pensées ${ }^{10}$. Cela revient en fait à poser la question de la définition de l'objet «ange» pour l'historien. Si c'est le terme de croyance qui est le plus souvent utilisé, Joad Raymond, qui ne s'interdit pas un usage provisoire du mot, objecte que l'on risque de réifier un système et propose donc de considérer les croyances sur les anges comme une forme de savoir (knowledge) «croisant et soutenant d'autres formes de savoir» politique ou philosophique, l'opération étant rendue possible justement par la présence des anges dans ces autres savoirs ${ }^{11}$. C'est d'ailleurs, il nous semble, un des intérêts majeurs de ces études et de l'étude de l'angélologie à l'époque moderne en général que de mettre, comme l'a fait Stuart Clark pour la démonologie ${ }^{12}$, la notion de savoir au cœur de l'enquête en

9. J. Raymond, Milton's angels, p. 39: «Angels appears in a broad array of works, from sermons to polemics to poems, and these writings did not simply reflect a pre-existing body of thought. [...] Modes of writing interacted with notions of angelic being and action »; cf. L. Sangha, Angels and belief, p. 107-108.

10. L. Sangha, Angels and belief, p. 3. Plus loin (p. 10), l'auteur ajoute: «Because they were theologically malleable, angels were recruited by clergymen of all stripes in support of their particular reformed stance ». Voir aussi p. 95 et p. 189. L'auteur se prévaut de Pierre Bourdieu.

11. J. Raymond, Milton's angels, p. 21 ; Id, «Introduction » à Conversation with angels, p. 2 : «Pre-1700 (and this date is open to debate and relocation) angels were more integrated into political theory, theology, social institutions, music, science, literary theory, and intellectual life generally, not only as content, but as a way of thinking about form and the thing itself ». Sur l'histoire des savoirs, voir Stéphane Van Damme (éd.), Histoire des sciences et des savoirs, t. 1, Paris, 2015 et Peter Burke, What is the history of knowledge ?, Cambridge, 2016.

12. Stuart Clark, Thinking with demons. The idea of witchcraft in early modern Europe, Oxford, 1997. L'auteur redonne à l'ensemble des discours et connaissances sur les démons, leur cohérence de savoirs en privilégiant un point de vue interne. Voir déjà Sophie Houdard, Les sciences du diable : quatre discours sur la sorcellerie, $\mathrm{XV}^{e}$-XVII ${ }^{e}$ siècles, Paris, 1988. 
l'étendant à des discours rarement pris en compte par l'histoire des sciences et des savoirs, comme l'est la théologie ${ }^{13}$.

Quelle signification donner alors à cette ubiquité des anges dans l'Angleterre moderne ? Est-elle le fruit d'une simple résistance des croyances catholiques aux changements doctrinaux et, pour reprendre l'expression lancée par Gerald Strauss et reprise pour l'Angleterre par Christopher Haigh, la manifestation d'un des «échecs» de la Réforme anglaise ${ }^{14}$ ? Ou au contraire, le témoignage de la vitalité d'une culture religieuse protestante capable de développer une angélologie fondée sur des principes exégétiques et doctrinaux propres ? L'historiographie avait déjà abordé la question. Robert West, dans un ouvrage classique sur les anges dans l'œuvre de Milton, remarquait déjà l'abondance des écrits sur les créatures spirituelles dans l'Angleterre du milieu du XVII ${ }^{\mathrm{e}}$ siècle, tout en laissant entendre que la volonté d'expurger la théologie de la présence des anges allait de soi, comme allait de soi le désintérêt des philosophes naturels pour la question ${ }^{15}$. Plus récemment, dans la perspective d'une histoire culturelle, on a avancé l'idée que les anges, en vertu de leur statut scripturaire assuré, se seraient substitués à des figures plus compromettantes. Jürgen Beyer a ainsi montré le remplacement de la Vierge Marie et des saints patrons par des figures angéliques dans les récits d'apparition de l'Europe luthérienne; Bruce Gordon a décrit comment les anges ont supplanté les âmes des défunts après que le rejet du purgatoire a rendu leur retour dans le monde des vivants impossible; de manière plus générale, Diarmaid MacCulloch a considéré que, dans le protestantisme, les anges ont pris la place

13. J. Raymond, introduction à Conversation with angels, p. 17: «To explore the internal coherence of faith is to risk writing something that sounds like theology or even apologia. Yet [...] it is necessary to possess a degree of imaginative sympathy in order to achieve comprehension. Perhaps, then, it is necessary to reverse the tendency of recent decades, among historians and especially among the literary critics, and write the history of religion in a way that sounds like theology ». Mais déjà Robert West, dans son ouvrage Milton and the angels (Athens, 1955) envisageait l'angélologie comme une science « intégrée » (p. 18).

14. Gerald Strauss, «Success and failure in the German Reformation », Past and Present, 67/1, 1975, p. 30-63 ; Christopher Haigh, « Success and failure in the English Reformation », Past and Present, 173/1, 2001, p. 28-49.

15. R. West, Milton and the angels, p. 21-22. Le dossier a été rouvert ensuite par Stephen Fallon dans Milton among the philosophers, Ithaca, 1991, p. 137-167. 
des saints catholiques dans le rôle d' " amis de l'humanité » plus adaptés « idéologiquement ${ }^{16}$.

La faiblesse de la thèse de la substitution, font remarquer nos auteurs, est de postuler la plus grande vitalité du catholicisme et d'envisager par conséquent la persistance des anges comme une concession à la faiblesse des «mentalités collectives » en cédant à une vision fonctionnaliste de la religion. Selon Raymond, il faut au contraire privilégier le point de vue interne et envisager la vitalité des anges à l'intérieur de l'ensemble de la culture protestante :

Le développement de la doctrine des anges en Grande-Bretagne après 1500 fut probablement davantage déterminé par des dynamiques religieuses et intellectuelles internes que par les réponses à la doctrine des anges du Catholicisme romain. Les anges protestants ne doivent pas être compris comme un résidu de la théologie antérieure à la Réformation. Les anges étaient vivants dans la culture, trop puissamment connectés à d'autres questions pour être réduits à une politique confessionnelle ${ }^{17}$.

La formulation indique que, pour Raymond, le point de vue interne est privilégié sans pour autant être exclusif. Son approche, et cela vaut aussi pour le travail de Sangha, donne une position éminente au discours théologique et doctrinal. Commençons par esquisser la position des fondateurs de la culture protestante, à savoir les réformateurs. Les anges ne sont pas leur préoccupation principale, le culte des saints, les indulgences ou la présence réelle étant des sujets autrement plus importants. De manière générale, l'angélologie de Luther, de Calvin et des premiers réformateurs

16. Jürgen Beyer, "Conceptions of holiness in the lutheran countries, c. 1550-1700», Studies in Folklore and Popular Religion, vol. 3, Papers delivered at the Symposium "Christian Folk Religion", éd. Ülo Valk, Tartu, 1999, p. 137-168 ; Bruce Gordon, «Malevolent ghosts and ministering angels : apparitions and pastoral care in the Swiss Reformation ", The Place of the dead: death and remembrance in late medieval and early modern Europe, éd. B. Gordon, P. Marshall, Cambridge, 2000, p. 87-109 ; Diarmaid MacCulloch, Reformation : Europe's house divided, 1490-1700, Londres, 2003, p. 8. Parmi les autres ouvrages abordant la thématique des anges, voir la synthèse d'Euan Cameron, Enchanted Europe : superstition, reason and religion, 1250-1750, Oxford, 2010 et certaines études réunies dans Clare Copeland et Jan Machielsen (éd.), Angels of light? Sanctity and the discernment of spirits in the early modern period, Leyde - Boston, 2013.

17. Dans le même sens L. Sangha, p. 51; A. Walsham et P. Marshall, introduction à leur volume Angels in the early modern world, p. 1-40; P. Marshall, «The guardian angel in Protestant England», Conversations with angels, éd. J. Raymond, p. 295-316. 
résultent de l'application du principe sola Scriptura mais aussi d'une réaction aux « excès » de la piété tardo-médiévale ${ }^{18}$. Compte tenu des innombrables mentions des anges dans les écritures, l'édifice angélologique était loin de devoir s'écrouler mais le nombre de développements fondés sur les écrits apocryphes, les Pères ou encore la philosophie grecque entraînait de fait des coupes claires dans le corpus. Il y a ainsi un rejet des «vaines » spéculations, notamment celles, inspirées du pseudo-Denys, relatives aux hiérarchies angéliques. Si, à la limite, les protestants acceptent l'idée de distinctions et d'un ordre parmi les anges, il n'est pas question d'aller au-delà de ce simple constat ${ }^{19}$. Le culte des anges doit en outre, comme celui des saints, être extirpé des pratiques religieuses. Enfin, les ministères et les offices des anges sont redéfinis afin de ne pas faire ombrage au seul médiateur, le Christ. Ils sont avant tout les agents de la providence divine et viennent souligner la toute-puissance de Dieu: les réformateurs ont ainsi largement accepté la figure de l'ange gardien des lieux et des royaumes dont le fondement scripturaire était par ailleurs incontestable. Si donc les anges ont gardé leurs traits traditionnels et s'ils ont rempli des fonctions assurées par les saints avant la Réforme, c'est surtout parce que leur existence était fondée sur l'Écriture. L'angélologie protestante n'est donc pas le résultat d'une «accommodation» de la doctrine catholique ni un simple « résidu » du catholicisme ${ }^{20}$.

$\mathrm{Si}$ cette construction doctrinale forme l'armature des développements angélologiques ultérieurs, elle n'a pas toujours été respectée au pied de la lettre. Il faut d'abord nuancer le respect du principe sola Scriptura. Le rejet des spéculations scolastiques par les réformateurs, de manière d'ailleurs plus ou moins virulente -Calvin les présentant comme une des voies menant droit à l'idolâtrie, Luther n'y voyant au contraire que de simples adiaphora, a priori inutiles mais pas forcément néfastes -, n'a pas

18. P. Marshall et A. Walsham (éd.), Angels in early modern world, p. 13 : «Angels had been badly compromised by their collaboation with many of the worst excesses of the late medieval devotional regime ».

19. Voir sur le sujet Feisal Mohamed, In the anteroom of Divinity. The Reformation of the angels from Colet to Milton, Toronto, 2008.

20. Laura Sangha utilise le terme d' « accommodation » là où Joad Raymond parle de « residuality ». 
empêché certains théologiens protestants de rédiger des traités d'angélologie similaires à ceux des catholiques et plus largement de traiter ponctuellement les principales questions qui préoccupaient les scolastiques depuis Pierre Lombard (création, nombre, connaissance, corps, langage $)^{21}$. Ensuite, la figure a été considérée avec beaucoup plus d'urgence au $\mathrm{XVII}^{\mathrm{e}}$ siècle qu'auparavant par les auteurs anglais, ces derniers la faisant déborder du cadre tracé par les réformateurs. On pourrait l'expliquer par une sorte de réaction à l'essor contemporain de la dévotion dans le catholicisme mais le terrain anglais, sans éliminer ce facteur confessionnel, invite surtout à y lire une réponse aux anxiétés d'une époque troublée et un enjeu de l'effervescence politique, religieuse et scientifique des deux derniers tiers du XVII ${ }^{\mathrm{e}}$ siècle anglais. Le traitement de la question doit passer par une analyse du temps long des croyances et par une prise en compte des ruptures censées les avoir réorganisées : la remise en cause du consensus élisabéthain et calviniste par Laud, les décennies révolutionnaires et l'Interrègne et enfin les troubles consécutifs à la Restauration monarchique. Cela passe aussi par la prise en compte de discours non exclusivement religieux : politiques, scientifiques et littéraires.

\section{L'ANGE DANS LA CULTURE \\ PROTESTANTE ANGLAISE DU XVII ${ }^{\text {E }}$ SIÈCLE}

Le développement d'une angélologie dans l'Angleterre d'après la Réforme est contemporain de l'émergence, selon Sangha, d'une «identité protestante anglaise distincte». Comment caractériser cette «culture protestante anglaise », parfois simplement qualifiée de «culture anglaise » ou de «culture protestante ${ }^{22}$ ? Il y a tout d'abord des caractéristiques théologiques propres à la réforme en général, que ce soit une modification de la sensibilité qui dévalue la vue et les arts visuels au profit de l'écoute et de l'écriture ${ }^{23}$, ou

21. Voir J. Raymond, Milton's angels, p. 48-88.

22. L. Sangha, Angels and belief, p. 79. Plus loin, Sangha parle d'une « angélologie anglaise » juste avant de la qualifier d' «angélologie protestante » (p. 95).

23. J. Raymond, Milton's angels, p. 5 : «British Protestants did little to create and circulate an alternative visual iconography of angels. They did, however, write about angels». Voir aussi A. Walsham, «Angels and idols in England's long 
une accentuation de la toute-puissance de Dieu ${ }^{24}$. Pour les anges cela signifie que si les apparitions angéliques sont réputées avoir cessé tout comme les miracles et les prophéties ${ }^{25}$, elles conservent néanmoins le rôle d'agents actifs de la providence. Il faut ensuite souligner le caractère pluraliste de la culture des Îles britanniques, en raison de la présence persistante de catholiques, de la grande variété des courants se réclamant de la réforme, du contexte des trois royaumes dans lesquels une majorité confessionnelle devait vivre avec des minorités majoritaires dans les deux autres royaumes et enfin de l'incapacité des gouvernements successifs à imposer l'uniformité. À ce titre, les anges ont joué un rôle dans « l'établissement de différences à l'intérieur du protestantisme $»^{26}$ à partir du règne de Charles $I^{\text {er }}$ et de la remise en cause, notamment par l'essor de l'arminianisme, du consensus élisabéthain, évolution où l'historiographie voit une des principales causes du déclenchement de la guerre civile ${ }^{27}$. C'est à la fois parce que les anges sont partout mais qu'ils ne sont pas pour autant un sujet central qu'ils pouvaient constituer un moyen pour exprimer des idées et des opinions divergentes. Enfin, le développement de la philosophie naturelle a suivi des voies en partie différentes de celles qu'elle a suivies dans l'Europe catholique et les anges ont trouvé une place dans cette réflexion épistémologique. Tous ces éléments interagissent dans un $\mathrm{XVII}^{\mathrm{e}}$ siècle anglais où la perméabilité entre théologie, littérature et science a été soulignée et où la guerre civile constitue, comme en témoigne la réflexion de Hobbes, une caisse de résonance.

Après une éclipse durant le règne d'Elisabeth $\mathrm{I}^{\text {re }}$ où l'attitude la plus commune relève plutôt de l'agnosticisme ${ }^{28}$, les anges sont

Reformation », Angels in the early modern world, dir. A. Walsham et P. Marshall, Cambridge, 2006, p. 134-167 et ead. «Invisible helpers », Past and Present, 2010, p. 105 où l'auteur remarque toutefois que la culture protestante « is no longer possible to describe as wholly iconophobic ». Sur la vision à l'époque moderne, voir S. Clark, Vanities of the eye: vision on early modern European culture, Oxford, 2007.

24. Pour l'Angleterre, voir A. Walsham, Providence in early modern England, Oxford, 1999.

25. A. Walsham, «Miracles in post-Reformation England», Studies in Church History, 41, 2005, p. 273-306.

26. J. Raymond, Milton's angels, p. 37.

27. N. Tyacke, Anti-calvinists : the rise of english arminianism c. 1590-1640, Oxford, 1987 et Peter White, Predestination, policy and polemic : conflict and consensus in the english church from the Reformation to the civil war, Cambridge, 1992.

28. J. Raymond, Milton's angels, p. 39. 
mobilisés au début du XVII ${ }^{\mathrm{e}}$ siècle pour soutenir les innovations portées par Laud dans les cérémonies - la présence des anges lors de la messe justifiant la tenue vestimentaire et la fonction du prêtre qui est souvent comparé aux créatures spirituelles - et dans la doctrine - l'essor de l'arminianisme libérant un espace pour l'invocation et la protection individuelle des anges gardiens. Le premier traité portant entièrement sur les anges composé par John Salked en 1613, par sa nature crypto-catholique - l'auteur est un ancien élève des collèges jésuites ibériques -, constitue de ce point de vue un des signes annonciateurs d'une réhabilitation qui touchent également les puritains et les non-conformistes ${ }^{29}$. Les bouleversements politiques et religieux à partir de 1640 donnent en effet aux anges, associés par les puritains au «romanisme $»^{30}$, une grande actualité polémique. L'illustration la plus éloquente de la réaction puritaine est sans doute l'ordonnance du Parlement de mai 1644 demandant de détruire, en plus des images de la Trinité, de la Vierge ou des saints, les représentations des anges dans les églises. Mais le rejet des images des anges, aussi spectaculaire qu'il puisse paraitre à l'historien à travers cette décision par ailleurs assez exceptionnelle ${ }^{31}$, ne signifie pas leur disparition de l'horizon culturel anglais, loin s'en faut: "Si la Réforme a fait paraître les anges plus éloignés de l'expérience quotidienne, ce fut le contraire durant les deux décennies révolutionnaires $»^{32}$. On voit alors se multiplier les écrits sur les anges ainsi que les témoignages attestant leur intervention dans la vie des hommes: récits de conversion, présages, communications angéliques, expériences visionnaires. Selon Raymond, cela s'explique par les tendances apocalyptiques, l'essor d'une théologie mystique et les angoisses relatives à «la fragmentation sociale et politique et à la guerre civile » mais aussi par la suppression de la censure qui ne révèle rien moins, selon lui, que le «développement d'un

29. John Salked, A Treatise of angels : of the nature, essence, place, power, science, will, apparitions, grace, sinne, and all other proprieties of angels, Printed by T.S... for Nathaniel Butter, Londres, 1613.

30. J. Raymond, Milton's angels, p. 38.

31. A. Walsham, «Angels and idols », p. 134 : «It was a subordinate issue, usually eclipsed by the more pressing question of whether or not it was permissible to portray the deity in any tangible form ».

32. J. Raymond, Milton's angels, p. 94. 
appétit populaire pour la théologie systématique $»^{33}$. Les anges sont utilisés comme figure «rhétorique » dans les libelles et les pamphlets pour dénoncer les adversaires religieux et politiques, la polémique anti-catholique s'attaquant en particulier à la doctrine de la garde individuelle et universelle qui devient parfois un véritable marqueur confessionnel. La multiplication des opinions sur les anges pourrait ainsi refléter cet état de fragmentation de la politique et de la société anglaise voire, comme le suggère Thomas Hobbes, l'avoir suscitée ${ }^{34}$.

Un des traits les plus remarquables de cette vitalité de l'angélologie durant les décennies révolutionnaires est l'élaboration d'une doctrine puritaine de l'ange gardien individuel en conformité avec la doctrine calviniste de la prédestination. Au contraire de l'individualité et de l'universalité de la garde angélique diffusées par le catholicisme, les auteurs puritains insistent sur l'idée que seuls les élus ont un ange gardien. On trouve cette présentation notamment dans deux longs traités exclusivement consacrés aux créatures spirituelles: An History of angells d'Henry Lawrence paru en 1646 rapidement considéré comme une «autorité protestante » en la matière, et en 1653 The Deputation of angels, or, the angell-guardian par Robert Dingley ${ }^{35}$. Ce dernier explique d'ailleurs l'écriture de son livre par la difficulté d'aborder le sujet auparavant en raison de l'esprit d'opposition à Rome qui régnait alors en Angleterre. Cela ne l'empêche pas de se prêter lui aussi au jeu en dressant la liste de toutes les erreurs catholiques en la matière. Sangha y voit la preuve qu'il s'agit bien d'une « réhabilitation » de l' angélologie, signe « d'une plus grande

33. Ibid., p. 100. L'auteur est un spécialiste des journaux et des pamphlets $\mathrm{du} \mathrm{XVII}^{\mathrm{e}}$ siècle : voir id., Pamphlets and pamphleteering in early modern Britain, Cambridge, 2003.

34. Dans Léviathan et la pompe à air: Hobbes et Boyle entre science et politique (Paris, 1993, p. 111), Steven Shapin et Simon Schaffer notent que : «L'ontologie dualiste déployée par les prêtres parlait de choses immatérielles: cela amenait les hommes à "voir double" et débouchait sur une fragmentation de l'autorité conduisant inévitablement au chaos et à la guerre civile. Les aristotéliciens parlaient d'essences séparées qui étaient instillées dans les entités matérielles; et les vacuistes peuplaient d'esprits les espaces qu'ils refusaient à la matière. Telles étaient les ressources ontologiques des ennemis de l'ordre».

35. J. Raymond, Milton's angels, p. 103 ; P. Marshall, « The guardian angel in protestant England », Conversations with angels, p. 295-316. 
assurance des protestants anglais $»^{36}$. Mais le lien entre l'élection et l'ange gardien n'apparaît plus après les années 1660 , ce qui montre son conditionnement par le contexte polémique des années révolutionnaires ${ }^{37}$.

Cette réflexion sur le rôle des anges auprès des élus s'accompagne d'un travail sur la figure de l'ange gardien des lieux et des communautés notamment chez Milton, Marwell, Wither auteurs de poèmes qui interrogent «l'état de la nation» anglaise dans cette époque bouleversée. Malgré l'opposition de certains puritains comme Andrew Willet, la doctrine était, on l'a vu, plus largement admise depuis les débuts de la Réforme. Le lien entre les deux figures est établi par le concept de providence : les peuples protégés par des anges sont aussi des peuples élus ${ }^{38}$. Mais la réflexion est aussi politique. Au milieu du siècle, l'évêque Joseph Hall écrit que «la vie des anges est politique, pleine de relations avec eux-mêmes et avec nous » et à la veille de la Glorieuse Révolution, John Scott, le chanoine de Saint Paul, qualifie d' «angelocracy » le gouvernement des hommes par les anges gardiens ${ }^{39}$. Joad Raymond voit dans le travail de Milton sur la figure de l'ange gardien de l'Angleterre dans son poème Lycidas, paru en 1637 et publié de nouveau en 1645 au début de la période révolutionnaire, un essai de définition de l' «englishness » débarrassée de la référence au roi. De même que la protection de saint Michel envers le peuple juif permet d'associer peuple et nation, de même la théologie réformée identifie la vraie Église avec les croyants plus qu'avec les institutions chargées de les encadrer. Cela suppose une relation entre la nation et le peuple qui ne repose pas sur l'autorité politique. L'ange, exécuteur de la providence, assure ce lien de voisinage et cette continuité historique de la nation anglaise que la disparition de l'institution monarchique ne remet pas en cause ${ }^{40}$. On comprend alors que la protection des anges ait pu être étendue aux Républiques et que le détenteur de

36. L. Sangha, Angels and belief, p. 109.

37. Ibid., p. 112.

38. A. Walsham, Providence in early modern England.

39. Cité par Raymond, Milton's angels, p. 19 et 87.

40. Ibid., p. 242 : «In other words, the local guardian angel presents a substitute, or a metonym, for a missing notion of nationhood. The doctrine articulates a sense of what it means to be providentially attached to, to be identified with, to be rooted in a place». 
l'autorité politique durant l'Interrègne, Olivier Cromwell, ait pu être comparé à l'ange gardien de l'Angleterre par le premier assistant de Milton, Andrew Marwell dans The first anniversary of the goverment under his Highness the Lord Protector paru en 1655. Ainsi, le principe posé par la pensée protestante d'une fin des apparitions et des miracles après l'avènement du Christ est nuancé par la nécessité de montrer l'action de la providence aux côtés des individus comme aux côtés des collectivités en lui donnant une agency qui paraisse humaine. Les anges peuplent donc les paysages de l'Angleterre de la première modernité, ce qui dément a priori le désenchantement que la Réforme est supposée avoir favorisé ${ }^{41}$.

L'inscription des anges dans l'ensemble de la culture et des savoirs anglais apporte d'autres enseignements. Premièrement, et dans le prolongement de ce que l'on vient de dire, l'angélologie protestante s'est adaptée aux nouvelles théories de la philosophie naturelle anglaise. Ainsi, placer les anges à proximité des hommes répond certes au besoin de protection mais aussi à la prise de conscience de l'élargissement des dimensions de l'univers. Même pour des anges se mouvant à la vitesse de mille miles à l'heure, comme le suppose par exemple William Heywood, les déplacements entre les cieux et la terre peuvent prendre des années. Il faut donc les rapprocher des hommes. Deuxièmement, certains représentants éminents de la philosophie naturelle comme Robert Boyle ou Richard Hooke précédés par d'autres personnalités comme le platonicien Henry More se sont servis de l'angélologie dans leur lutte contre le matérialisme et l'athéisme ${ }^{42}$ incarnés surtout par la philosophie de Thomas Hobbes, mais dont la filiation englobe Descartes et Gassendi. Ils l'ont fait en prouvant l'existence des anges par la collecte de récits d'interventions et d'apparitions angéliques, qui relève d'une pratique de l'enquête qui doit beaucoup à la méthodologie empirique de la philosophie naturelle ${ }^{43}$. Enfin, le XVII ${ }^{e}$ siècle ne

41. Voir à ce sujet A. Walsham, The reformation of the landscape. Religion, identity, and memory in early modern Britain and Ireland, Oxford, 2011.

42. La qualification est polémique et recouvre en fait aussi bien des positions athées proprement dites que celles des déistes ou des Sociniens.

43. L. Sangha, Angels and belief, p. 169-186; J. Raymond, Milton's angels, p. 277-310 ; A. Walsham, «Invisible helpers », p. 122-129. 
clôt pas la saison de l'angélologie contrairement à ce que pensait Robert West qui lie cette disparition des anges à l'essor de la science moderne ${ }^{44}$. Les anges continuent à occuper à la fin du siècle et au début du suivant une place éminente dans les réflexions épistémologiques des plus grands penseurs de l'époque. Comme le montre Simon Schaffer dans une contribution passionnante, la formulation par Newton du principe de la gravitation universelle qui débouche sur la publication des Principia mathematica en 1687 s'accompagne d'une réflexion intense sur les anges et les esprits qu'il imagine peupler les espaces interstellaires. Newton est loin d'être un cas isolé et ses contemporains ou disciples comme Edmond Halley, Richard Bentley, William Whiston ou Daniel Defoe peuplent eux aussi le nouveau cosmos d'esprits et d'anges afin de prouver à l'encontre du mécanicisme et de ses implications athées l'existence du non-matériel. Au même titre, l'intelligence angélique continue à être présentée à la fois comme un modèle et une limite pour l'intelligence humaine: l'instrumentation scientifique - notamment le télescope - permet ainsi de restaurer les capacités d'avant la chute que les anges n'ont, à la différence des hommes, pas perdues. N'imaginons pas ces discussions limitées aux publications scientifiques et aux échanges épistolaires. Comme durant les décennies révolutionnaires, elles investissent l'espace public, la cour, la chaire, Newton étant notamment présenté par ses partisans comme un ange transitant à travers les cieux ou conversant avec les êtres spirituels ${ }^{45}$. Toutefois, cette appropriation de l'objet ne va pas sans modification du langage utilisé pour décrire les anges, qui se détache progressivement des connotations précisément théologiques.

La présence des anges dans les différents savoirs n'est pas seulement la conséquence de leur statut théologique, philosophique ou scientifique particulier. Les anges fonctionnent en fait comme des figures de passage : non seulement l'objet passe

44. R. West, Milton and the angels, p. 21 : «In England as elsewhere, angelology died of mere disuse some time near the end of the seventeenth century. As beings with almost no function left in nature, angels could no longer claim attention of the able, and the "science" of them passed out of all serious prominence, whether in literature, philosophy, or religion ».

45. Simon Schaffer, «Newtonian angels», Conversations with angels, p. $90-122$. 
d'un savoir à un autre mais avec lui circulent les questionnements, comme le montre l'épopée de Milton qui ouvre des perspectives à la fois sur l'optique, la physique, la vitesse ou le nombre des anges ${ }^{46}$. Une des raisons en est l'ambiguïté constitutive de la figure de l'ange dans des écrits où se mêlent l'utilisation figurative - comme dispositif narratif ou rhétorique - et sa présentation littérale - les anges chez Milton mangent, chantent, dorment, font l'amour... ${ }^{47}$ Or cette ambiguïté renvoie de manière plus générale à la question de l'accommodation, c'est-à-dire au processus par lequel les vérités divines ineffables sont communiquées aux capacités limitées de l'intellect humain dans l'Écriture ${ }^{48}$. C'est ici que la réflexion de Raymond se montre la plus profonde même si sa portée reste problématique ou en tout cas à mesurer. Dans le protestantisme, selon Raymond, l'accent mis sur la sola Scriptura aurait rendu possible une interprétation figurative à l'intérieur d'une configuration littéraliste. En outre, l'accommodation, même après la Réforme, s'est étendue à d'autres discours, notamment celui des prophètes dont la voix ne s'est pas tue dans l'Angleterre du XVII ${ }^{\mathrm{e}}$ siècle. Or, soutient Raymond, Milton se revendique un poète divinement inspiré. C'est la raison pour laquelle les anges sont si nombreux dans son poème. L'omniprésence des anges dans Le Paradis perdu n'est ni simplement littérale ni simplement métaphorique, elle est les deux à la fois et illustre en quelque sorte le chevauchement de la doctrine et de la fiction, de la théologie et de la poésie en montrant que la littérature peut être productrice de connaissance, de savoir. Cela conduit Raymond à envisager ce point en inscrivant les anges dans une histoire de l'imagination. Les anges sont un fondement de l'imagination moderne au sens où ils fondent la possibilité de représenter quelque chose, et on pourrait ajouter ici qu'ils sont en quelque sorte, en suivant l'analyse de Castoriadis, un des «schèmes organisateurs » de

46. J. Raymond, Milton's angels, p. 309: «While he [Milton] was doing this, inserting angels within, or inserting within angels an understanding of natural philosophy, natural philosophers were taking up questions that had previously belonged to theologians, and theologians were responding to or drawing upon natural philosophy. These were importantly permeable boundaries ».

47. J. Raymond, Milton's angels, p. 267-276.

48. Ibid., «Introduction », p. $4:$ « representation must conform to theological truths $[\ldots]$ and theology relies upon representation (in particular, a theory of how the ineffable can be comprehended by the limited faculties of humans) ». 
l'imaginaire d'une sociétée ${ }^{49}$. En considérant la question à travers le poème et les écrits de Milton, Raymond n'entend pas pour autant considérer l'œuvre de Milton comme représentatif ou typique de la culture anglaise mais plutôt comme un exemple parmi d'autres voix tour à tour ou simultanément poétique, prophétique et scientifique qui partageaient certains attendus concernant les êtres spirituels.

\section{DÉSENCHANTEMENT, RÉENCHANTEMENT :}

\section{ANGÉLOLOGIE PROTESTANTE ET ANGÉLOLOGIE CATHOLIQUE}

La Réforme et l'essor des sciences expérimentales ont donc mené, dans la culture anglaise, non pas à un effacement progressif des anges mais au contraire à une revitalisation de l'angélologie que Raymond n'hésite pas à rapprocher de la revitalisation de l'angélologie scolastique par l'aristotélisme à partir du XIII ${ }^{\mathrm{e}}$ siècle. Il ne faut donc pas se représenter une théologie rigide ou morte dans l'Angleterre du XVII ${ }^{\mathrm{e}}$ siècle face à une science conquérante ${ }^{50}$. Certes la Réforme a bien entraîné un déclin de la représentation visuelle des anges et de leur présence dans le culte et les prières et désormais le lien entre les hommes et les anges passe «par les mots et les idées» et non plus «par les images et les gestes $»^{51}$. Mais ce que montrent ces études est que le vide laissé par ceux-ci a pu être comblé par une intensification de l'utilisation de ceux-là, avec entre autres pour effet d'opérer un iconoclasme par «excessive visualisation $»^{52}$.

Sangha estime que l'ubiquité des anges dans la culture anglaise confirme la difficulté de se représenter la Réforme comme un processus linéaire de désenchantement. Elle rejoint ici la proposition d'Alexandra Walsham qui a envisagé le paradigme wéberien comme un modèle cyclique de désenchantement et de réenchantement, les deux derniers tiers du XvII ${ }^{\mathrm{e}}$ siècle

49. Cornelius Castoriadis, L'institution imaginaire de la société, Paris, 1975, p. 203. Voir aussi L. Sangha, Angels and belief, p. 4.

50. Voir John H. Brooke, Science and religion. Some historical perspectives, Cambridge, 1991, p. 22-68.

51. J. Raymond, Milton's angels, p. 356.

52. Ibid., p. 364. 
correspondant à un "partiel réenchantement du monde » alors que la période suivante se caractériserait par une sécularisation - assimilée par l'auteur au désenchantement ${ }^{53}$. Elle n'en dessine pas moins des lignes de fuite, comme lorsqu'elle postule une «psychologisation » progressive des anges au long du XVII ${ }^{\mathrm{e}}$ siècle. Au contraire, Raymond remet en cause de manière plus radicale les grands récits de la modernisation, que ce soit le désenchantement, la sécularisation qui lui est plus ou moins assimilée, ou celui plus proprement littéraire théorisé par Thomas Eliot d'une «dissociation de la sensibilité » qui se serait produite quelque part au milieu du $\mathrm{XVII}^{\mathrm{e}}$ siècle $^{54}$. Adoptant une approche plus décidément archéologique que généalogique, il leur préfère l'idée d'une succession «d'alliances ». Ainsi, l'alliance entre doctrine théologique et philosophie naturelle, à laquelle il s'intéresse plus particulièrement, "pourrait » s'être défaite quelque part dans les années 1740. Si d'autres alliances lui ont succédé, l'auteur l'admet, elles n'ont plus eu la même capacité intégrative et en définitive les «différentes perspectives ou différents langages sur les êtres et les actions angéliques se sont éloignés les uns des autres $»^{55}$. Il nous semble que la formulation a ici une valeur surtout provisoire et qu'elle invite à la prudence, même si ailleurs l'auteur paraît plaider plus simplement pour un report du processus plutôt que pour sa remise en cause. On est proche ici de l'emploi du terme de «migration » par Marshall et Walsham qui, pour signifier les

\section{L. Sangha, Angels and belief, p. 191-192.}

54. J. Raymond, Milton's angels, p. 365 et p. 381 où la thèse est particulièrement forte : «I am suggesting that some of the dichotomies conventionally discovered in the seventeenth century - Renaissance-Modernity, enchantment-secularization, God-Man, theory-history, apprehension-comprehension [...] are not supported by an analysis of the fortune of angels in early modern imaginative writing, and that if these dichotomies cannot be supported by an analysis of these beings that subtend across such broad areas of knowledge, belief and practice, then they did not operate in the way that is presently believed».

55. J. Raymond, «Introduction », p. 13. Voir la conclusion de la contribution de P. Marshall («The guardian angel in protestant England», p. 295-316, ici p. 312) dans ce même volume: "Across a century and more, the belief in individual guardian angels was that rare thing in a profoundly propositional and instinctively conformable age - a genuine matter of opinion [...]. Over the period, we can also discern a broad pattern whereby discussions of the guardian angel were becoming less a matter of theological probity or doctrinal correctness, and more a means of exploring ideas about providence and fortune, human physiology and personal psychology ». 
déplacements de sens, présente l'avantage de ne pas spécifier de direction particulière ${ }^{56}$.

Il peut alors être utile de confronter la situation anglaise à celle du catholicisme en nous demandant en quoi une approche en termes d'histoire culturelle peut apporter à la compréhension de l'angélologie catholique et ce que la présence des anges peut nous dire du désenchantement du monde dans le catholicisme. Remarquons d'emblée qu'on ne dispose pas d'études qui envisagent de manière aussi systématique la place des anges dans les savoirs et la littérature du catholicisme, l'évidence de leur ubiquité étant sans doute plus décourageante que leur supposée disparition dans le protestantisme. Pour l'historien du catholicisme toutefois, ces études semblent conforter l'idée avancée naguère par Bob Scribner, qu'entre protestants et catholiques la différence est de degré plus que de nature ${ }^{57}$. De même qu'il n'y pas de rejet de l'angélologie par le protestantisme mais un nouveau façonnement en fonction d'un contexte doctrinal propre, de même l'angélologie catholique n'est pas restée inchangée par-delà le concile de Trente. D'abord, la continuité revendiquée par l'Église catholique n'a pas empêché l'évolution et la réorganisation des savoirs théologiques ni leur interaction avec les débats scientifiques du moment ${ }^{58}$. D'autre part, comme dans le protestantisme, la question du salut est essentielle pour comprendre certains des aspects les plus significatifs de l'angélologie catholique. Sans doute de telles similitudes devraient-elles inviter à souligner davantage la circulation des écrits entre les deux pans de la chrétienté latine. Comme le remarquent les deux auteurs, un des points de départ de cette «renaissance» angélologique du $\mathrm{XVII}^{\mathrm{e}}$ siècle anglais est de manière significative l'ouvrage d'un catholique devenu anglican, John Salked, qui étudia à Coïmbre et à Cordoue et fut l'assistant de Suárez et de Vásquez. L'office liturgique inséré dans le bréviaire romain en 1608 est traduit en anglais en 1623. Enfin, le

56. P. Marshall et A. Walsham, «Migration of angels in the early modern world », Angels in the early modern world, p. 1-40. L'expression est empruntée à John Bossy, Christianity in the West 1400-1700, Oxford, 1985.

57. Bob Scribner, «The Reformation, popular magic and the "disenchantment of the world" ", Journal of Interdisciplinary History, 23, 1993, p. 475-94.

58. Voir, pour les implications de la querelle de auxiliis dans le champ des savoirs, Michael John Gorman, "Molinist theology and natural knowledge », Sciences et religions de Copernic à Galilée (1540-1610), Rome, 1999, p. 235-254. 
best-seller de la littérature de dévotion consacré à l'ange gardien, l'Horologium auxiliaris tutelaris angeli du jésuite Jérémie Drexel publié pour la première fois en latin en 1622, parait à son tour en anglais dès 1630 et n'a pas été lu uniquement par des catholiques ${ }^{59}$. Il reste évidemment des différences. Ainsi, l'ubiquité des anges dans le catholicisme n'est pas seulement textuelle, elle est également visuelle.

Montrer toutes les insuffisances des grands récits téléologiques de la modernisation occidentale est plus aisé que de s'en débarrasser totalement. Qu'il n'y ait pas eu d'évolution linéaire, on en conviendra et d'ailleurs Weber donne au processus du désenchantement un statut similaire à celui de l'idéal-type ${ }^{60}$. En ce qui concerne l'approche culturelle du religieux, elle pose, pour le catholicisme, la question de l'échelle d'analyse. Une entrée possible pour parcourir ce continent angélologique et ces lignes de faille est d'envisager la figure de l'ange gardien dont l'essor est contemporain de la réforme catholique ${ }^{61}$. Cette approche présente l'intérêt de pouvoir articuler doctrine et pratique autour du paradigme de la confessionnalisation en partant des textes officiels - catéchisme, office liturgique - pour envisager ensuite l'éventail des discours propres à l'ensemble de la culture confessionnelle catholique. Nous avons proposé l'idée que cet essor doit se lire à la lumière des controverses sur la grâce et sur le salut des infidèles, l'ange gardien permettant d'articuler la nature et la surnature, le libre arbitre et la grâce dont la distinction a toujours été problématique. C'est même dans la mesure où il ne peut $\mathrm{y}$ avoir de distinction claire à ce sujet que le détour par la figure de l'ange gardien a pu être proposé, principalement par des auteurs accentuant le rôle du libre arbitre, notamment les jésuites. L'ange gardien accordé à tous les hommes individuellement est comme l'assurance que l'initiative humaine concrétisée dans la réalisation

59. A. Walsham, «Catholic Reformation and the cult of angels in early modern England », Conversations with angels, p. 273-294.

60. François-André Isambert, «Le "Désenchantement du monde" : non sens ou renouveau du sens », Archives de sciences sociales des religions, 61-1, 1986, p. 83-103.

61. C'est ce que nous tentons de faire dans notre thèse, «L'ange gardien à l'époque moderne : culte, élaboration doctrinale et usages. $\mathrm{XVI}^{\mathrm{e}}-\mathrm{XVII}^{\mathrm{e}}$ siècle ", soutenue à l'EHESS le 19 décembre 2013, à paraitre aux éditions Les Belles Lettres. 
d'actions moralement bonnes peut mener au salut, y compris aux dires des plus optimistes, pour des païens totalement ignorants de la foi chrétienne. Mais l'ange gardien ne fonctionne pas seulement comme une figure permettant d'articuler théoriquement deux propositions antinomiques, l'action de la grâce de Dieu et la liberté humaine. Il permet de construire des expériences de pensée - la prière mentale, la direction spirituelle et intérieure, l'écriture spirituelle - dont le fondement doctrinal n'apparaît pas sûr ou du moins pas absolument clair. Il est l'un des agents de leur expression littéraire dans des ouvrages qui sont tout à la fois spirituels, dévotionnels et théologiques. En outre, l'ange gardien agit ici comme une figure de passage entre des savoirs et des pratiques mais aussi, une fois passée cette saison assez courte finalement de floraison de cette littérature, comme une figure ayant rendu possible leur éloignement les uns des autres. De ce point de vue, l'essor de l'ange gardien dans la dévotion et la spiritualité est un révélateur des « turbulences » plus générales que Pierre-Antoine Fabre a repérées dans les rapports entre littérature, spiritualité et théologie à partir du début du XVII ${ }^{\mathrm{e}}$ siècle $^{62}$.

C'est peut-être à partir de ce constat que l'on peut revenir à la question du désenchantement du monde. L'hypothèse dans le catholicisme est relativement négligée par la recherche qui s'est davantage attachée à comprendre les mécanismes sociaux de sécularisation et de déchristianisation. Michel de Certeau, qui n'utilise qu'avec précaution les concepts de sécularisation et de désenchantement, a cependant présenté l'essor de la figure de l'ange gardien comme un des symptômes de «l'émiettement» des grandes synthèses médiévales ou des «éclats des alliances théologiques », manifestation précoce du «christianisme éclaté » qu'il avait diagnostiqué quelques années auparavant ${ }^{63}$. Des historiens de l'Inquisition ont émis l'hypothèse que les tribunaux de la foi ont pu par exemple contribuer à ce processus, notamment

62. Pierre-Antoine Fabre, «Sciences sociales et histoire de la spiritualité moderne : perspectives de recherche », Recherches de science religieuse, 97/1, 2009, p. 33-51.

63. Michel de Certeau, «Le parler angélique. Figures pour une poétique de la langue ", Actes sémiotiques - Documents, 54, 1984, repris dans La fable mystique, t. 2, Paris, 2013 ; id. et Jean-Marie Domenach, Le christianisme éclaté, Paris, 1974. 
dans l'examen des dossiers de béatification, alors que la discipline interne des ordres religieux a pu mener au même résultat ${ }^{64}$. Les manifestations visuelles de l'ange gardien font ainsi l'objet de censures. Toutefois, les censeurs et les inquisiteurs ne font ici que se conformer aux textes fondateurs de la confession catholique comme le catéchisme tridentin qui met en garde contre la recherche des manifestations extraordinaires, certes sans les exclure a priori, comme le faisaient les théologiens protestants en postulant leur cessation depuis la venue du Christ. L'ubiquité iconographique pourrait également plaider en faveur du maintien plus fort d'un enchantement dans le catholicisme. Mais la multiplication des images peut avoir des effets ambivalents et on peut songer par exemple à l'hypothèse d'Alphonse Dupront d'une émancipation des anges de leur lieu de résidence céleste par le biais de la perspective dans l'art renaissant et baroque ${ }^{65}$.

antoine_mazurek@yahoo.it

64. Voir pour l'Italie, Adriano Prosperi, « L'elemento storico nelle polemiche sulla santità », Finzione e santità tra medioevo ed età moderna, éd. Gabriella Zarri, Turin, 1991, p. 88-118 ; pour l'Espagne, Andrew Keitt, « The Spanish Inquisition, and the disenchantment of the world », Journal of the History of Ideas, 65/2, 2004, p. 231-250.

65. Alphonse Dupront, Du sacré. Croisades et pèlerinages. Images et langages, Paris, 1987, p. 172. 